\title{
ON THE SPHERICAL SUMMABILITY OF MULTIPLE ORTHOGONAL SERIES
}

\author{
BY \\ JOSEPHINE MITCHELL
}

1. Introduction. Multiple orthogonal systems arise naturally in the following way. Let $E$ be the Cartesian product of $q$ measurable sets $E_{1}, \cdots, E_{q}$, where each $E_{k}$ may be assumed to be embedded in one-dimensional Euclidean space, although the dimensionality of the underlying space seems to be immaterial to our proofs. Let $\left\{\phi_{n}^{(\mathbf{k})}\right\}(n=0,1,2, \cdots ; k=1, \cdots, q)$ be a complete orthonormal set (ONS) of functions of class $L^{2}$ defined on the set $E_{k}$, that is,

(i) $\int_{E_{k}} \phi_{m}^{(k)}(x) \phi_{n}^{(k)}(x) d x=\delta_{m n}$ (ON relations) and

(ii) $\int_{E_{k}} \phi_{m}^{(k)}(x) f(x) d x=0(m=0,1,2, \cdots)$ for a function $f$ of class $L^{2}$ implies that $f=0$ almost everywhere (a.e.) on $E_{k}$ (completeness).

Then the set $\left\{\phi_{m_{1} \cdots m_{q}}=\phi_{m_{1}}^{(1)} \cdots \phi_{m_{q}}^{(q)}\right\}\left(m_{k}=0,1,2, \cdots ; k=1, \cdots, q\right)$ is a complete ONS on $E$. From this ONS a multiple orthogonal series $\sum a_{m_{1} \cdots m_{q}} \phi_{m_{1} \cdots m_{q}}$ is formed, where $\left\{a_{m_{1} \cdots m_{q}}\right\}$ is an arbitrary sequence of real constants such that $\sum a_{m_{1}}^{2} \cdots m_{q}<\infty{ }^{(1)}$, so that, by the Riesz-Fischer theorem extended to more variables, $a_{m_{1}} \cdots_{m_{q}}$ are the Fourier coefficients of a function $f$ of class $L^{2}$ with respect to the given ONS.

Following Bochner [3] $\left({ }^{2}\right)$ we sum the above orthogonal series by spherical partial sums $\left({ }^{3}\right)$ instead of the more usual rectangular partial sums. Thus a multiple orthogonal series is said to be convergent if

$$
\lim _{N_{k} \rightarrow \infty} \sum_{\nu \leqq N_{k}} \sum_{\nu=m_{1}+\cdots+m_{q}} a_{m_{1}} \cdots m_{q} \phi_{m_{1}} \cdots m_{q}
$$

exists, where $\left\{N_{k}\right\}$ is the sequence of non-negative integers which can be represented as the sum of $q$ squares of integers. We shall also have occasion to refer to convergence by rectangles (or rectangular convergence)- a multiple orthogonal series is convergent by rectangles if

$$
\lim _{M_{1} \ldots M_{q} \rightarrow \infty} \sum_{m_{1}=0}^{M_{1}} \cdots \sum_{m_{q}=0}^{M_{q}} a_{m_{1} \cdots m_{q}} \phi_{m_{1}} \ldots m_{q}
$$

exists.

Presented to the Society, December 28, 1950; received by the editors November 24, 1950.

(1) The summation for these two series is $\left(m_{k}=0\right.$ to $\left.\infty ; k=1, \cdots, q\right)$ but we omit the summation as long as the context is clear without it.

(2) Numbers in brackets refer to the references cited at the end of the paper.

(3) This method of summation has been found to be efficient in dealing with the convergence and summability of multiple Fourier series. Our results show that it is fully as efficient in analogous considerations for multiple orthogonal series. 
In considering (1.1) it is convenient to study the sequence $\left\{S_{N}\right\}$ $(N=0,1,2, \cdots)$, where

$$
S_{N}=\sum_{v=0}^{N} 0_{v}
$$

and $0_{\nu}=\sum_{\nu=\Sigma_{m}} a_{m} \phi_{m}\left(\sum_{m}=m_{1}^{2}+\cdots+m_{q}^{2} ; m=m_{1} \cdots m_{q}\right)$ or 0 according as $\nu$ can or cannot be represented as the sum of $q$ squares of integers. Then limit (1.1) equals $\sum_{\nu=0}^{\infty} 0_{\nu}$. Similarly the series $\sum a_{m}^{2}$ equals $\sum_{\nu=0}^{\infty} A_{\nu}$, where $A_{\nu}=\sum_{\nu=\Sigma_{m}} a_{m}^{2}$ if $\nu=\sum_{m}$ or 0 if $\nu \neq \sum_{m}$.

If we use the notation $(1.3)$, the sequence $\left\{S_{N}\right\}$ is $(C, 1)$ summable if $\lim _{N \rightarrow \infty} \sigma_{N}$ exists, where

$$
\sigma_{N}=\frac{S_{0}+\cdots+S_{N}}{N+1}=\sum_{\nu=0}^{N}\left(1-\frac{\nu}{N+1}\right) 0_{\nu}
$$

or, more generally, $\left\{S_{N}\right\}$ is $(C, \alpha)$ summable if $\lim _{N \rightarrow \infty} \sigma_{N}^{\alpha}$ exists, where

$$
\sigma_{N}^{\alpha}=\left(p_{N}^{\alpha}\right)^{-1} \sum_{\nu=0}^{N} p_{N \rightarrow p}^{\alpha} 0_{\nu} \quad(\alpha \neq-1,-2, \cdots)[9]
$$

and $p_{N}^{\alpha}=(\alpha+1) \cdots(\alpha+N) / N$ !

Instead of $(C, \alpha)$ summability Bochner and others $[3 ; 4 ; 5 ; 6]$ have considered Riesz mean summability of order $\delta$ and type $\nu((R, \nu, \delta)$ summability). The orthogonal series $\sum a_{m} \phi_{m}$ is $(R, \nu, \delta)$ summable if

$$
\lim _{R \rightarrow \infty} \sum_{\nu \leqq R}\left(1-\frac{\nu}{R}\right)^{\delta} 0_{\nu} \quad(0<R<\infty)(\delta>0)
$$

exists. However for our methods of proof we find it more convenient to study $(C, \alpha)$ summability and then use the fact that $(C, \alpha)$ and $(R, \nu, \alpha)$ summability are equivalent for arbitrary simple series [9].

In this paper we are able to generalize without difficulty the main theorems on convergence, absolute convergence, and $(C, 1)$ summability of simple orthogonal series to $q>1$. In $\$ 2$ we discuss the rôle of the Lebesgue functions in the convergence of multiple orthogonal series. In $\S 3$ several sufficient conditions are proved for the convergence and absolute convergence of multiple orthogonal series as well as a sufficient condition for divergence and some comparison is made with convergence by rectangles. In $\$ 4$ several sufficient conditions are given for the $(C, 1)$ summability of a multiple orthogonal series as well as sufficient condition for divergence. Also, analogously to the case $q=1$, we are able to prove the important result that $(C, 1)$ and $(C, \alpha)(\alpha>0)$ summability are equivalent for multiple orthogonal series. All of these results are exact generalizations both in statement and proof to the analogous theorems for simple orthogonal series. Basically this is due 
to the fact that the methods of proof used for simple orthogonal series depend mainly on certain properties of arbitrary simple series plus the orthogonality relation expressed in the form $a_{m}^{(k)} a_{n}^{(k)} \int_{E_{k}} \phi_{m}^{(k)} \phi_{n}^{(k)} d x=a_{m}^{(k) 2}(n=0,1,2, \cdots)$. For $q>1$, using spherical summation, a corresponding orthogonal relation holds, namely,

$$
\begin{aligned}
\sum_{\nu=\Sigma_{m}} \sum_{\mu=\Sigma_{n}} a_{m} a_{n} \int_{E} \phi_{m} \phi_{n} d x & =\sum_{\nu=\Sigma_{m}} a_{m}^{2} \\
\left(n_{k}\right. & =0,1,2, \cdots ; k=1, \cdots, q)(4),
\end{aligned}
$$

and the same properties of simple series are applicable. Indeed the results of this paper would be valid for any method of summation which reduces to a consideration of simple sequences, for example summation by diagonals, $\nu=m_{1}+\cdots+m_{q}$, as long as an orthogonality relation of form (1.7) holds, where, of course, $\sum_{m}$ would be replaced by some other functions of $m_{1}$ $\cdots, m_{q}$.

It is interesting to compare our results with those obtained both for rectangular summation of multiple orthogonal series and spherical summation of multiple Fourier series. For rectangular summation an $\mathrm{ON}$ relation similar to (1.7) holds but the properties of simple series may no longer be used. Also, if one uses the analogous conditions to those obtained for $q=1$, the proofs become very difficult and long [1] and in order to get simpler proofs more stringent hypotheses must be imposed [13]. In addition in the case of rectangular summation all generalizations, so far, are for $q=2$ and would become increasingly complicated for $q>2$, whereas for spherical summation the value of $q$ is immaterial. The difference between the proofs in the two cases lies in the fact that in the case of rectangular summation certain "cross-product" terms enter which are not present for $q=1$ or spherical summation. This is illustrated in the case of multiple Fourier series in that for spherical summability the behavior of the function need only be known in the neighborhood of the point $P$ considered, whereas for rectangular summation the behavior of the function must be known in a "cross-neighborhood" of $P$. In comparison with the sufficient conditions used in spherical convergence and summability of multiple Fourier series $[4 ; 5]$ our conditions will be seen to be much sharper. Also, our conditions for $(C, \alpha)$ summability apply to all $\alpha>0$ and are freed from the usual restriction $\alpha>(q-1) / 2$, which would seem to indicate that this requirement belongs to the method of proof and is not intrinsic to the method of summability. Of course, as in the case of simple orthogonal series our sufficient conditions suffer from a certain liability in that necessarily they are not based on the properties of the function $f$, which is to be developed, but rather either on the properties of the sequence $\left\{a_{m}\right\}$ or on the properties of the ONS $\left\{\phi_{m}\right\}$; also, our results apply only to functions of class $L^{2}$.

(4) Here $d x=d x_{1} \cdots d x_{q}$ is the Euclidean volume element on $E$ and $\int_{E}=\int_{E_{1}} \cdots \int_{E_{q}}$. 


\section{The spherical Lebesgue functions.}

2.1. Properties of the spherical Lebesgue function [cf. 12]. As in the case of rectangular summation the spherical Lebesgue function is defined by

$$
L_{N}(P)=\int_{E}\left|K_{N}(P, Q)\right| d x
$$

where $K_{N}(P, Q)$ is the kernel function of the ONS

$$
K_{N}(P, Q)=\sum_{\nu=0}^{N} \Phi_{\nu}(P, Q),
$$

$\Phi_{\nu}(P, Q)=\sum_{\Sigma_{m}=\nu} \phi_{m}(P) \phi_{m}(Q)$ or 0 according as $\nu=\sum_{m}$ or $\nu \neq \sum_{m}$.

As for the case $q=1$ if the ONS $\left\{\phi_{m}\right\}$ is bounded on $E$, then $L_{N}(P)=O\left(N^{q / 4}\right)$.

Proof. By (2.1) and the Schwarz inequality

$$
L_{N}^{2}(P)=O\left(\int_{E}\left\{\sum_{\nu=0}^{N} \Phi_{\nu}(P, Q)\right\}^{2} d x\right) .
$$

Using the ON relations as in (1.7), we get

$$
L_{N}(P)=O\left(\sum_{\nu \leqq N} \sum_{\nu=\Sigma_{m}} \phi_{m}^{2}(P)\right)=O\left(\sum_{\nu \leqq N} \sum_{\nu=\Sigma_{m}} 1\right)=O\left(N^{q / 2}\right),
$$

since the last sum is the number of lattice points in the hypersphere $m_{1}^{2}+\cdots+m_{q}^{2} \leqq N$, which equals $O\left(N^{q / 2}\right)$ [16]. Thus the conclusion follows.

Again for any ONS of class $L^{2}$, as for the case $q=1, L_{N}(P)$ is $\left.o\left(N^{q / 4}\right)(\log N)^{(1+\epsilon) / 2}\right)$ for arbitrary positive $\epsilon$.

Proof. The series of positive terms $S=\sum^{\prime}\left(m_{1}^{2}+\cdots+m_{q}^{2}\right)^{-q / 2}$ - $\left[\log \left(m_{1}^{2}+\cdots+m_{q}^{2}\right)\right]^{-1-\epsilon}$ is convergent for arbitrary positive $\epsilon\left(^{5}\right)$, which may be shown in the following way. From the inequality $\left(m_{1}^{2}+\cdots+m_{q}^{2}\right)$ $\geqq q^{-1}\left(m_{1}+\cdots+m_{q}\right)^{2}$ results $\left(m_{1}^{2}+\cdots+m_{q}^{2}\right)^{-q / 2}\left[\log \left(m_{1}^{2}+\cdots+m_{q}^{2}\right)\right]^{-1-\bullet}$ $\leqq q^{q / 2}\left(m_{1}+\cdots+m_{q}\right)^{-q}\left[\log q^{-1}\left(m_{1}+\cdots+m_{q}\right)^{2}\right]^{-1-\epsilon}$. Furthermore the series of positive terms $\sum^{\prime}\left(m_{1}+\cdots+m_{q}\right)^{-q}\left[\log q^{-1}\left(m_{1}+\cdots+m_{q}\right)^{2}\right]^{-1-\epsilon}$ is convergent, for, summing it along $m_{1}+\cdots+m_{q}=\nu$, we get $\sum_{\nu=1}^{\infty} \nu^{-q}\left(\log q^{-1} \nu^{2}\right)^{-1-\epsilon} \sum_{\nu=m_{1}+\cdots+m_{q}} 1$; but $\sum_{\nu=m_{1}+\cdots+m_{q}} 1$ is $O\left(\nu^{q-1}\right)$ and $\nu^{2} q^{-1} \geqq \nu$ for $\nu \geqq q$ so that the preceding series is $O\left(\sum_{\nu=1}^{\infty} \nu^{-1}(\log \nu)^{-1-\epsilon}\right)$ and this latter series converges. Thus the series $S$ also converges.

[For $q \geqq 5$ a more direct proof of the convergence of series $S$ is the following. Since the terms of the series are positive, $S=\sum_{\nu=2}^{\infty} \nu^{-q / 2}(\log \nu)^{-1-\epsilon} \sum_{\nu=\Sigma_{m}} 1$. For $q \geqq 5$ the number of lattice points in the hypersphere $m_{1}^{2}+\cdots+m_{a}^{2} \leqq \nu$ is $\alpha_{q} \nu^{q / 2}+O\left(\nu^{q / 2-1}\right)$, where $\alpha_{q}$ depends only on $q$ [16], so that the number of lattice points on $m_{1}^{2}+\cdots+m_{q}^{2}=\nu$ is at most $O\left(\nu^{q / 2-1}\right)$. Thus $S=O\left(\sum^{\prime} \nu^{-1}(\log \right.$ $\left.\nu)^{-1-\epsilon}\right)=O(1)$.]

By the ON relations

(5) The sign ' indicates that meaningless terms are omitted. 


$$
\begin{aligned}
\sum_{\nu=2}^{\infty} \nu^{-q / 2}(\log \nu)^{-1-\epsilon} \int_{E} \Phi_{\nu} & (P, P) d x \\
& =\sum\left(m_{1}^{2}+\cdots+m_{q}^{2}\right)^{-q / 2}\left[\log \left(m_{1}^{2}+\cdots+m_{q}^{2}\right)\right]^{-1-\epsilon} \\
& =O(1),
\end{aligned}
$$

and $\sum_{\nu=2}^{\infty} \nu^{-q / 2}(\log \nu)^{-1-\epsilon} \Phi_{\nu}(P, P)<\infty$ a.e. in $E$. By a Kronecker theorem, $K_{N}(P, P)=\sum_{\nu=0}^{N} \Phi_{\nu}(P, P)$ is $o\left(N^{q / 2}(\log N)^{1+\epsilon}\right)$ and, since the proof of the first property shows that $L_{N}^{2}(P)=O\left(K_{N}(P, P)\right)$, the conclusion follows.

REMARK. For bounded ONS the bound for $L_{N}(P)$ is analogous to that obtained for the rectangular Lebesgue function $L_{N_{1} \cdots N_{q}}(P)$, namely $O\left(\left[\left(N_{1}+1\right) \cdots\left(N_{q}+1\right)\right]^{1 / 2}\right)$, since $\left(N_{1}+1\right) \cdots\left(N_{q}+1\right)$ is the number of lattice points in the parallelepiped $\left(0 \leqq m_{k} \leqq N_{k}, k=1, \cdots, q\right)$ and $O\left(N^{q / 2}\right)$ is the order of the number in the hypersphere $m_{1}^{2}+\cdots+m_{q}^{2} \leqq N$.

2.2. The Lebesgue functions and spherical convergence of multiple orthogonal series. Using the same method of proof as for $q=1$ we get an analogous result $[11]$.

THEOREM 2.1. Let $\sum a_{m} \phi_{m}$ be the orthogonal development of a function $f$ of class $L^{2}$ with respect to the complete ONS $\left\{\phi_{m}\right\}$. If $L_{N}(P)=O\left(u^{2}(N)\right), 0$ $<u(N) \leqq u(N+1)$, then the sequence $\left\{u^{-1}(N) S_{N}(P)\right\}$ approaches a limit a.e. in $E$. In particular if the Lebesgue functions are bounded, the orthogonal development of $f$ is a.e. convergent on $E$.

Proof. Set $v_{N}=\max _{0 \leqq j \leqq N} u^{-1}(j) S_{j}=u^{-1}(p) S_{p}$, where $p$ depends on $P$ and $N$. The sequence $\left\{v_{N}\right\}$ is monotonic nondecreasing and we show that $I_{N}$ $=\int_{E} v_{N} d x_{P}=O(1)$, from which it follows that $\lim _{N \rightarrow \infty} v_{N}$ exists a.e. on $E$ and the sequence $\left\{u^{-1}(k) S_{k}\right\}$ is bounded above a.e. on $E$. By Fubini's theorem and the Schwarz inequality

$$
\begin{aligned}
I_{N}^{2} & =\left\{\int_{E} d x_{P} \int_{E} f(Q) K_{p}(P, Q) u^{-1}(p) d x_{Q}\right\}^{2} \\
& =O\left(\int_{E} d x_{Q}\left(\int_{E} K_{p}(P, Q) u^{-1}(p) d x_{P}\right)^{2}\right) .
\end{aligned}
$$

By the ON properties $\int_{E} K_{p}(P, Q) K_{p^{\prime}}\left(P^{\prime}, Q\right) d x_{Q}=K_{t}\left(P, P^{\prime}\right)$, where $t$ $=\min \left(p, p^{\prime}\right)$, so that

$$
\begin{aligned}
\int_{E} d x_{Q}\left(\int_{E} K_{p}(P, Q)\right. & \left.u^{-1}(p) d x_{P}\right)^{2} \\
& =\int_{E} d x_{Q} \int_{E} \int_{E} K_{p}(P, Q) K_{p^{\prime}}\left(P^{\prime}, Q\right) u^{-1}(p) u^{-1}\left(p^{\prime}\right) d x_{P} d x_{P^{\prime}} \\
& =\int_{E} \int_{E} K_{t}\left(P, P^{\prime}\right) u^{-1}(p) u^{-1}\left(p^{\prime}\right) d x_{P} d x_{P^{\prime}},
\end{aligned}
$$


and from the given hypotheses

$$
\begin{aligned}
I_{N}^{2}= & O\left(\int_{E} \int_{E}\left|K_{t}\left(P, P^{\prime}\right)\right| u^{-1}(p) u^{-1}\left(p^{\prime}\right) d x_{P} d x_{P^{\prime}}\right) \\
= & O\left(\int_{E} u^{-2}(p) d x_{P} \int_{E}\left|K_{p}\left(P, P^{\prime}\right)\right| d x_{P^{\prime}}\right. \\
& \left.+\int_{E} u^{-2}\left(p^{\prime}\right) d x_{P^{\prime}} \int_{E}\left|K_{p^{\prime}}\left(P, P^{\prime}\right)\right| d x_{P}\right) \\
= & O(1) .
\end{aligned}
$$

Since similarly $\left\{u^{-1}(N) S_{N}\right\}$ is bounded below a.e., $\lim \sup _{N \rightarrow \infty}\left|u^{-1}(N) S_{N}\right|$ is finite a.e. for each $f \in L^{2}$ on $E$.

In order to show that $\lim _{N \rightarrow \infty} u^{-1}(N) S_{N}$ exists a.e. on $E$ we show that the remaining hypotheses of a well known theorem on linear functionals due to Banach [2] are satisfied, namely: If a sequence $\left\{U_{N}(X)\right\}$ of functionals, linear and continuous in measure, is such that (i) $\lim \sup U_{N}(X)$ is finite a.e. for each $X$, (ii) $\lim U_{N}(X)$ exists a.e. for each $X$ belonging to a set $B$ everywhere dense in the domain $E$, in which $U_{N}$ are defined, then the sequence $\left\{U_{N}(X)\right\}$ approaches for each $X$ a.e. a functional, linear and continuous in measure. In our case the sequence $U_{N}(X)=u^{-1}(N) S_{N}(P)$, where $X=f$, is linear and continuous in measure and we have only to show that $\lim _{N \rightarrow \infty} u^{-1}(N) S_{N}$ exists a.e. for each $f$ belonging to a set $B$ everywhere dense in $E$ with respect to the metric, $\int_{E} f^{2} d x$, of $L^{2}$. But this follows from the completeness of the ONS. For let $g=\sum_{\mu \leqq M} \sum_{\mu=\Sigma_{n}} c_{n} \phi_{n}$, where $\left\{c_{n}\right\}$ is an arbitrary finite set of constants. Then $S_{N}(g)=\sum_{p \leqq N} \sum_{\nu=\Sigma_{m}} a_{m} \phi_{m}$, where $a_{m}=\sum_{\mu \leqq M} \sum_{\mu=\Sigma_{n}} c_{n} \int_{E} \phi_{n} \phi_{m} d x=c_{m}$, so that $S_{N}(g)=\sum_{\nu \leqq \min (N, M)} \sum_{\nu=\Sigma_{m}} c_{m} \phi_{m}$ and $\lim _{N \rightarrow \infty} S_{N}(g)$, being a finite sum, exists and equals $S_{M}(g)$, so that $\lim _{N \rightarrow \infty} u^{-1}(N) S_{N}(g)$ also exists. Also the set $\{g\}$ is everywhere dense in $E$ with respect to the metric of $L^{2}$, that is, given $f \in L^{2}$ and $\epsilon>0$, there exists a $g=\sum_{p \leqq M} \sum_{p=\Sigma_{n}} c_{n} \phi_{n}$ such that $\int_{E}(f-g)^{2} d x<\epsilon$, since this relation follows from the completness of the ONS [cf. the proof of Theorem 355 in 12].

\section{3 . Convergence of the multiple orthogonal series.}

3.1. Theorem 3.1. If the series $\sum^{\prime} \log ^{2} \nu a_{m}^{2}<\infty$, then the multiple orthogonal series $\sum a_{m} \phi_{m}$ is convergent a.e. on $E$.

Proof. Again the proof for $q=1$ may be generalized [cf. 12].

(i) The convergence of $\sum^{\prime} \log \nu a_{m}^{2}$ implies that $S_{2^{k}}$ approaches a limit a.e. in $E\left({ }^{6}\right)$. Suppose that $f$ is the function of class $L^{2}$ whose Fourier coefficients equal $a_{m}$. Then, using Parseval's relation, $\sum a_{m}^{2}=\int_{E} f^{2} d x$, and the ON properties, we get

(6) It is convenient to use logs to the base 2 in our proofs. 


$$
\int_{E}\left(f-S_{2^{k}}\right)^{2} d x=\sum_{\nu=2^{k}+1}^{\infty} A_{\nu} \equiv r_{2^{k}} .
$$

From Abel's transformation it follows that

$$
\begin{aligned}
\sum_{k=0}^{\infty} r_{2^{k}} & =\sum_{k=0}^{\infty}(k+1)\left(r_{2^{k}}-r_{2^{k+1}}\right)+\lim _{k \rightarrow \infty}(k+1) r_{2^{k+1}} \\
& =\sum_{k=0}^{\infty}(k+1) \sum_{\nu=2^{k+1}}^{2 k+1} A_{\nu}+\lim _{k \rightarrow \infty}(k+1) \sum_{\nu>2^{k+1}} A_{\nu} \\
& <\sum_{k=0}^{\infty} \sum_{\nu=2^{k}+1}^{2 k+1} \log 2 \nu A_{\nu}+\lim _{k \rightarrow \infty} \sum_{\nu>2^{k+1}} \log 2 \nu A_{\nu} \\
& <2 \sum^{\prime} \log \nu a_{m}^{2}<\infty .
\end{aligned}
$$

Thus the series $\sum_{k=0}^{\infty}\left(f-S_{2}\right)^{2}<\infty$ a.e. on $E$ and $S_{2}{ }^{k} \rightarrow f$ a.e. on $E$.

(ii) It remains to show that $\sum^{\prime} \log ^{2} \nu a_{m}^{2}<\infty$ implies that $\lim _{N \rightarrow \infty}\left(S_{2}{ }^{k}-S_{N}\right)$ $=0$ a.e. on $E$ for $\left(2^{k}<N<2^{k+1}\right)$. We use the following lemma due to Rademacher [15]: Let $S\left(\alpha, \alpha^{\prime}\right)=\sum_{\nu=\alpha+1}^{\alpha \prime} c_{\nu}$. Then for all $N$ such that $2^{k}<N<2^{k+1}$, $S^{2}\left(2^{k}, N\right) \leqq k \sum_{\left(\alpha, \alpha^{\prime}\right)} S^{2}\left(\alpha, \alpha^{\prime}\right)$, where $\left(\alpha, \alpha^{\prime}\right)$ is summed over all number pairs of the form $\left(2^{k}+h 2^{j}, 2^{k}+h 2^{j}+2^{j-1}\right)\left(j=1, \cdots, k ; h=0,1, \cdots, 2^{k-j}-1\right)$ $\left(2^{k}<\alpha<\alpha^{\prime} \leqq 2^{k+1}\right)$. (We note that Rademacher states this result for simple orthogonal series but his proof depends only on the dyadic representation of the subscripts, plus the Schwarz inequality.)

Using this lemma and the $\mathrm{ON}$ relations we get

$$
\begin{aligned}
\int_{E}\left(S_{N}-S_{2^{k}}\right)^{2} d x & \leqq k \int_{E} \sum_{\left(\alpha, \alpha^{\prime}\right)}\left(\sum_{\nu=\alpha+1}^{\alpha^{\prime}} 0_{\nu}\right)^{2} d x=k \sum_{\left(\alpha, \alpha^{\prime}\right)} \sum_{\nu=\alpha+1}^{\alpha^{\prime}} A_{\nu} \\
& \leqq k^{2} \sum_{\nu=2^{k}+1}^{2 k+1} A_{\nu}
\end{aligned}
$$

where the last inequality follows from the fact that each sum $\alpha<\nu \leqq \alpha^{\prime}$ includes not more terms then $2^{k}<\nu \leqq 2^{k+1}$. Also this last sum is less than or equal to $\sum_{\nu=2^{k+1}}^{2^{k+1}} \log ^{2} \nu A_{\nu}$, so that

$$
\sum_{k=0}^{\infty} \int_{E}\left(S_{N}-S_{2^{k}}\right)^{2} d x \leqq \sum^{\prime} \log ^{2} \nu a_{m}^{2},
$$

and the series $\sum_{k=0}^{\infty}\left(S_{N}-S_{2}{ }^{k}\right)^{2}<\infty$ a.e. on $E$, from which the desired conclusion follows.

3.2. Counter-examples. Agnew [1] has proved that for $q=2$ the convergence of the series $\sum \log ^{2}(m+1) \log ^{2}(n+1) a_{m n}^{2}$ implies that the corresponding double orthogonal series is convergent by rectangles a.e. We now use a theorem on simple orthogonal series in order to construct counterexamples which show that this condition and the condition imposed in 
Theorem 3.1 cannot be improved upon for $q>1$.

TheOREM 3.2. (i). If $0 \leqq v\left(m_{1}, \cdots, m_{q}\right)=o\left(\log ^{2}\left(m_{1}^{2}+\cdots+m_{q}^{2}\right)\right)$ and $v\left(m_{1}, \cdots, m_{q}\right)$ is monotonic nondecreasing with respect to each $m_{k}$, there exists an ONS $\left\{\phi_{m}\right\}$ and a sequence $\left\{a_{m}\right\}$ such that $\sum_{m_{1}, \cdots, m_{q}=0}^{\infty} v\left(m_{1}, \cdots, m_{q}\right) a_{m}^{2}$ $<\infty$ but $\sum_{\nu=0}^{\infty} 0_{\nu}=\infty$ everywhere on a measurable set $E$.

(ii). If $0 \leqq u\left(m_{1}, \cdots, m_{q}\right)=o\left(\log ^{2}\left(m_{1}+2\right) \cdots \log ^{2}\left(m_{q}+2\right)\right)$ and $u\left(m_{1}, \cdots, m_{q}\right)$ is monotonic nondecreasing with respect to each $m_{k}$, then there exists an ONS $\left\{\psi_{m}\right\}$ and a sequence of numbers $\left\{b_{m}\right\}$ such that $\sum b_{m}^{2} u\left(m_{1}, \cdots, m_{q}\right)<\infty$ but $\sum_{m_{1}, \ldots, m_{q}=0}^{\infty} b_{m} \psi_{m}=\infty$ everywhere on a measurable set $E^{\prime}$.

Proof. Call $w\left(m_{q}\right)=v\left(0, \cdots, 0, m_{q}\right)$. Then $w\left(m_{q}\right)=o\left(\log ^{2} m_{q}\right)$ and by Theorem 5.37 [12] there exists an ONS $\left\{\phi_{m_{q}}\right\}$ and a sequence of numbers $\left\{a_{m_{q}}\right\}$ such that $\sum_{m_{q}=0}^{\infty} a_{m_{q}}^{2} w\left(m_{q}\right)<\infty$ but $\sum_{m_{q}=0}^{\infty} a_{m_{q}} \phi_{m_{q}}=\infty$ everywhere on a measurable set $E_{1} \subset$ Euclidean $E^{1}$ space. Setting $\phi_{m}=\phi_{m_{1}} \cdots \phi_{m_{q}}$ defines an ONS on the measurable set $E=E_{1} X \cdots X E_{1} \subset E^{q}$. Let $a_{0} \cdots m_{q}=a_{m_{q}}$ for $m_{q}=0,1,2, \cdots$, and $a_{m_{1} \cdots m_{q}}=0$ otherwise. Then $\sum a_{m}^{2} v\left(m_{1}, \cdots, m_{q}\right)$ $=\sum_{m_{q}=0}^{\infty} a_{m_{q}}^{2} w\left(m_{q}\right)<\infty$ whereas $\sum_{\nu=0}^{\infty} 0_{\nu}=\left(\phi_{0}\right)^{q-1} \sum_{m_{q}=0}^{\infty} a_{m_{q}} \phi_{m_{q}}=\infty$ everywhere on $E$.

In order to prove (ii), we set $w^{\prime}\left(m_{q}\right)=u\left(0, \cdots, 0, m_{q}\right)$ so that $w^{\prime}\left(m_{q}\right)$ $=o\left(\log ^{2}\left(m_{q}+2\right)\right)$ and proceed as in the proof of (i).

3.3. It is of some interest to discuss the connection between spherical and rectangular convergence. In the case of absolute convergence both methods, of course, are equivalent for arbitrary multiple series but, as is shown below, for conditionally convergent series neither method implies the other; however in the case of multiple orthogonal series rectangular summation does seem to require a stronger condition for convergence, at least for $q=2$, than that necessary for spherical convergence.

In the first place there exist ONS which converge spherically but not by rectangles. Since $\log \left(m_{1}^{2}+\cdots+m_{q}^{2}\right)=o\left(\log m_{1} \cdots \log m_{q}\right)$, in Theorem 3.2 (ii) we can take $u\left(m_{1}, \cdots, m_{q}\right)=\log ^{2}\left(m_{1}^{2}+\cdots+m_{q}^{2}\right)$. Then there exists an ONS $\left\{\phi_{m}\right\}$ and a number sequence $\left\{a_{m}\right\}$ such that $\sum a_{m}^{2} \log ^{2}\left(m_{1}^{2}+\cdots\right.$ $\left.+m_{q}^{2}\right)<\infty$ but $\sum_{m_{1}, \ldots, m_{q}=0}^{\infty} a_{m} \phi_{m}=\infty$ everywhere on a measurable set $E$. On the other hand by Theorem 3.1 the series $\sum a_{m} \phi_{m}$ converges spherically a.e. on $E$.

Moreover there exist series which converge by rectangles but not spherically as the following example shows-the proof depending upon the fact that the rectangular partial sums of a convergent multiple series need not be bounded but the spherical partial sums (since they form a simple sequence) are necessarily bounded. Let $\sum u_{m}$ be a convergent multiple series of positive terms and $\left\{v_{n}\right\}$ an unbounded simple sequence of positive terms. Form the series $\sum u_{m}^{\prime}$, where $u_{0, \ldots, 0 m_{q}}^{\prime}=u_{0} \ldots o m_{q}+v_{m_{q}}, u_{10}^{\prime} \ldots o m_{q}$ $-v_{m_{q}}\left(m_{q}=0,1,2, \cdots\right)$, otherwise $u_{m}^{\prime}=u_{m}$. Obviously $\sum_{\mu_{1}, \ldots, \mu_{q}=0}^{m_{1}, \ldots, m_{m}}$ 
$=\sum_{\mu_{1}, \ldots, \mu_{q}=0}^{m_{1}, \ldots, m_{q}} u_{m}$ for $m_{1} \geqq 1$ and $m_{k} \geqq 0(k>1)$ so that the multiple series $\sum u_{m}^{\prime}$ converges by rectangles. On the other hand the spherical partial subsequence $S_{n}^{2}=\sum_{\nu \leqq n}{ }^{2} \sum_{v=\Sigma_{m}} u_{m}+v_{n}^{2}$ is divergent so that $\lim _{N \rightarrow \infty} S_{N}$ cannot exist.

3.4. Absolute convergence. We have the following theorem.

THEOREM 3.3. The convergence of the series $\sum\left|a_{m}\right|^{2-\epsilon}$ for $0<\epsilon<2$ implies the absolute convergence a.e. of the series $\sum a_{m} \phi_{m}$.

Proof. We order the ONS $\left\{\phi_{m}\right\}$ into a simple sequence $\left\{\phi_{p}\right\}$ by any arbitrary method, for example, by diagonals. The sequence $\left\{\phi_{p}\right\}$ still forms an ONS. Also, on account of the absolute convergence, $\sum\left|a_{m}\right|^{2-\epsilon}=\sum_{p=0}^{\infty}\left|a_{p}\right|^{2-\epsilon}$. By Theorem 5.42 [12] for simple ONS the series $\sum_{p=0}^{\infty} a_{p} \phi_{p}$ converges a.e. and it is true for every ordering. Thus the series $\sum_{p=0}^{\infty} a_{p} \phi_{p}$ converges absolutely e.a., that is, the series $\sum a_{m} \phi_{m}$ converges absolutely a.e., since the order of summation is immaterial for absolutely convergent series.

Sufficient conditions analogous to the hypotheses in Theorems 5.41 and 5.43 [12] could also be obtained in a similar manner.

Remarks. (i) We must note that Theorem 5.42 (depending on Theorem 5.41 and Lemma 5.34) is proved for a one-dimensional set only but the proof appears to be independent of the dimension of the set.

(ii) We may favorably compare Theorem 3.3 with a result due to Chandresekaharan [4] for multiple Fourier series, namely, $\sum v^{q / 2+\epsilon}\left|a_{m}\right|^{2}$ $<\infty, \epsilon>0$, implies the existence of $\lim _{R \rightarrow \infty} \sum_{\nu \leqq R}\left|\sum_{\nu=\Sigma_{m}} a_{m} e^{i\left(m_{1} x_{1}+\cdots+m_{q} x_{q}\right)}\right|$.

3.5. It seems to me that we could obtain an interesting sufficient condition for the (spherical) convergence of multiple orthogonal series based on the properties of the function $f$, whose development is given by the orthogonal series in question, by generalizing a method due to Helly for $q=1$ [10]. If $f$ is assumed to be of bounded variation in the sense of Vitali (or possibly of Fréchet) so that the multiple Stieltjes integral of $f$ with respect to continuous functions (or functions with jumps along a finite number of hyperplanes) exists and an integration by parts formula is available, then the orthogonal development of $f$ will converge if the ONS satisfies certain conditions analogous to the Helly conditions. In this connection we mention certain new conditions due to Morse and Transue for rectangular convergence of multiple Fourier series [14].

4. The spherical summability of multiple orthogonal series.

4.1. Necessary and sufficient condition for $(C, 1)$ summability. As is proved in $\$ 4.4$ it is sufficient to consider $(C, 1)$ summability. Thus we have the following theorem.

THEOREM 4.1. A necessary and sufficient condition that a multiple orthogonal series $\sum a_{m} \phi_{m} b e(C, 1)$ summable is that the sequence $\left\{S_{2^{k}}{ }^{k}\right.$ converge a.e. on $E$.

Proof. Following the method of proof for $q=1$ [12] we set $\sigma_{N}-S_{2}{ }^{k}$ 
$=\left(\sigma_{N}-S_{2}{ }^{k}\right)+\left(\sigma_{2^{k}}-S_{2^{k}}\right)$ and consider the two terms in parentheses on the right side. Now

$$
\sigma_{N}-S_{N}=-\sum_{v=0}^{N} \frac{\nu}{N+1} 0_{v}
$$

Also, by the $\mathrm{ON}$ relations

$$
\sum_{k=0}^{\infty}\left(2^{k}+1\right)^{-2} \int_{E}\left(\sum_{\nu=0}^{2 k} \nu 0_{\nu}\right)^{2} d x=\sum_{k=0}^{\infty}\left(2^{k}+1\right)^{-2} \sum_{\nu=0}^{2 k} \nu^{2} A_{\nu}
$$

and this latter sum equals

$$
\sum_{v=1}^{\infty} \nu^{2} A_{\nu} \sum_{k=E[\log \nu]}^{\infty}\left(2^{k}+1\right)^{-2} \leqq \frac{4}{3} \sum a_{m}^{2}\left({ }^{7}\right) .
$$

Hence the series $\sum_{k=0}^{\infty}\left(2^{k}+1\right)^{-2}\left(\sum_{\nu=0}^{2^{k}} \nu 0_{v}\right)^{2}$ converges a.e. in $E$ and $\lim _{k \rightarrow \infty}\left(\sigma_{2^{k}}-S_{2}{ }^{k}\right)=0$ a.e. in $E$.

It remains to show that $\lim _{N \rightarrow \infty}\left(\sigma_{N}-\sigma_{2^{k}}\right)=0$ a.e. $\left(2^{k}<N<2^{k+1}\right)$. Now

$$
\sigma_{N}-\sigma_{2^{k}}=\sum_{r=2^{k}}^{N-1}\left(\sigma_{r+1}-\sigma_{v}\right)
$$

and by the Schwarz inequality

$$
\left(\sigma_{N}-\sigma_{2^{k}}\right)^{2} \leqq \sum_{v=2^{k}}^{2 k+1-1} \nu\left(\sigma_{v+1}-\sigma_{v}\right)^{2}
$$

since $\sum_{\nu=2^{k}}^{2^{k+1}-1} \nu^{-1} \leqq 1$. Also,

$$
\sigma_{\nu+1}-\sigma_{\nu}=\sum_{\alpha=0}^{\nu+1} \frac{\alpha}{(\nu+1)(\nu+2)} 0_{\alpha}
$$

and, if we use the ON properties,

$$
\int_{E}\left(\sigma_{\nu+1}-\sigma_{\nu}\right)^{2} d x=\sum_{\alpha=0}^{\nu+1} \frac{\alpha^{2}}{(\nu+1)^{2}(\nu+2)^{2}} A_{\alpha} .
$$

Thus

$$
\begin{aligned}
\sum_{\nu=0}^{\infty} \nu \int_{E}\left(\sigma_{\nu+1}-\sigma_{\nu}\right)^{2} d x & \leqq \sum_{\nu=0}^{\infty} \frac{1}{(\nu+1)(\nu+2)^{2}} \sum_{\alpha=0}^{\nu+1} \alpha^{2} A_{\alpha} \\
& \leqq \sum_{\alpha=1}^{\infty} \alpha^{2} A_{\alpha} \sum_{\nu=\alpha-1}^{\infty}(\nu+1)^{-3} \\
& \leqq \sum a_{m}^{2}<\infty
\end{aligned}
$$

(7) The symbol $E[\log \nu]$ means the nearest integer greater than or equal to $\log \nu$. 
Therefore $\sum_{\nu=0}^{\infty} \nu\left(\sigma_{\nu+1}-\sigma_{\nu}\right)^{2}<\infty$ a.e. on $E$. Hence $\lim _{k \rightarrow \infty} \sum_{\nu=2^{k}}^{2^{k+1}} \nu\left(\sigma_{\nu+1}-\sigma_{\nu}\right)^{2}$ $=0$ a.e. on $E$, and the theorem follows.

4.2. Sufficient condition for $(C, 1)$ summability. As for the case $q=1$ we have the following theorem.

THEOREM 4.2. The convergence of the series $\sum^{\prime}(\log \log \nu)^{2} a_{m}^{2}$ implies the $(C, 1)$ summability of the multiple orthogonal series $\sum a_{m} \phi_{m}$ a.e. on $E$.

Proof. From Theorem 4.1 it is sufficient to show the convergence of the sequence $\left\{S_{2^{k}}\right\}$ a.e.

In the first place $\sum^{\prime} \log \log \nu a_{m}^{2}<\infty$ implies that $S_{2^{2}}$ approaches a limit a.e. on $E$. Using the notation $r_{2}{ }^{k}$ introduced in $\$ 3.1$ and Abel's transformation, we have as in $\$ 3.1$

$$
\sum_{p=0}^{\infty} r_{2^{2^{p}}}=\sum_{p=0}^{\infty}(p+1) \sum_{\nu=2^{2^{2 n}}+1}^{2^{2 p+1}} A_{\nu}+\lim _{p \rightarrow \infty}(p+1) \sum_{\nu>2^{2^{p+1}}} A_{\nu}
$$

and, since $p+1=\log \log 2^{2^{p+1}}<\log \log 4 \nu$ for $\nu>2^{2^{p}}$, the left side is less than or equal to

$$
\sum_{p=0}^{\infty} \sum_{\nu=2^{2^{p}}+1}^{2^{p+1}} \log \log 4 \nu A_{\nu}+\lim _{p \rightarrow \infty} \sum_{\substack{\nu=2^{2 p+1}+1 \\ 2}}^{\infty} \log \log 4 \nu A_{\nu}<2 \sum \log \log \nu a_{m} .
$$

Thus as in the proof of Theorem 3.1, $S_{2^{2}} \rightarrow f$ a.e. on $E$.

It remains to show that $\sum^{\prime}(\log \log \nu)^{2} a_{m}^{2}<\infty$ implies that $\lim _{p \rightarrow \infty}\left(S_{2^{k}}-S_{2^{2}}\right)$ $=0$ a.e. on $E\left(2^{2^{p}}<2^{k}<2^{2^{p+1}}\right)$. Following the method of proof given by Rademacher for $q=1$ [15], let the dyadic representation of $k$ be $\sum_{v=0}^{p} \theta_{\nu} 2^{p-\nu}$ $\left(\theta_{\nu}=0\right.$ or 1$)$ and break up the sum $\sum_{\nu=2^{2}+1}^{2^{k}}$ into $p$ sums

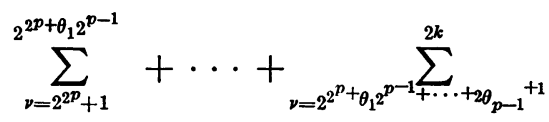

(those terms, whose last $\theta_{\nu}$ is 0 , are replaced by 0 ). Then using the lemma quoted in $\S 3.1$, where here $\left(\alpha, \alpha^{\prime}\right)$ is summed over number pairs of the form $\left(2^{2^{p}+\mu 2^{\nu}}, 2^{2^{p}+\mu 2^{\nu}+2^{p-1}}\right)\left(\nu=1, \cdots, p ; \mu=0,1, \cdots, 2^{p-\nu}-1 ; 2^{2^{p}}<\alpha, \alpha^{\prime} \leqq 2^{2^{p+1}}\right)$ so that there are the same number of pairs $\left(\alpha, \alpha^{\prime}\right)$ as in the lemma (with $k$ replaced by $p$ ), and the ON relations, we have as on p. 142

$$
\begin{aligned}
\int_{E}\left(S_{2^{k}}-S_{2^{2^{p}}}\right)^{2} d x & \leqq p \int_{E} \sum_{\left(\alpha, \alpha^{\prime}\right)} S^{2}\left(\alpha, \alpha^{\prime}\right) d x \\
& =p \int_{E} \sum_{\left(\alpha, \alpha^{\prime}\right)}\left(\sum_{\nu=\alpha+1}^{\alpha^{\prime}} 0_{\nu}\right)^{2} d x \\
& =p \sum_{\left(\alpha, \alpha^{\prime}\right)} \sum_{\nu=\alpha+1}^{\alpha^{\prime}} A_{\nu} \leqq p^{2} \sum_{\nu=2^{2 p}+1}^{2^{2 p+1}} A_{\nu}
\end{aligned}
$$


Hence

$$
\begin{aligned}
\sum_{p=1}^{\infty} \int_{E}\left(S_{2^{k}}-S_{2^{2}}\right)^{2} d x & =\sum_{p=1}^{\infty} p^{2} \sum_{\nu=2^{2^{p}}+1}^{2^{2 p+1}} A_{\nu} \\
& \leqq \sum_{p=1}^{\infty} \sum_{\nu=2^{2^{p}}+1}^{2^{2 p+1}}(\log \log \nu)^{2} A_{\nu} \\
& =\sum^{\prime}(\log \log \nu)^{2} a_{m}^{2},
\end{aligned}
$$

from which the theorem follows.

4.3. Counter-examples to $(C, 1)$ (spherical) and $(C, 1, \cdots, 1)$ (rectangular) summability. For the case $q=1$ if $0 \leqq v(n)=o\left((\log \log n)^{2}\right), \lim v(n)=\infty$, $v(n)$ is nondecreasing and $\sum_{n=0}^{\infty} b_{n}^{2} v(n)<\infty$, then there exists an orthogonal series $\sum_{n=0}^{\infty} b_{n} \psi_{n}(t)$ which is summable $(C, 1)$ at no point of $[0,1][12]$.

If we set $b_{m}=b_{m_{1}} \cdots b_{m_{q}}$, then $\sum v\left(m_{1}\right) \cdots v\left(m_{q}\right) b_{m}^{2}<\infty$. Let $\psi_{m}$ $=\psi_{m_{1}} \cdots \psi_{m_{q}}$. Also

$$
\begin{aligned}
\sigma_{m} & =\left(m_{1}+1\right)^{-1} \cdots\left(m_{q}+1\right)^{-1} \sum_{\nu_{1}=0}^{m_{1}} \cdots \sum_{\nu q=0}^{m_{l}} S_{\nu} \\
& =\prod_{k=1}^{q}\left[\left(m_{k}+1\right)^{-1} \prod_{\nu_{k}=0}^{m_{k}} S_{\nu_{k}}\right] \\
& =\prod_{k=1}^{q} \sigma_{m_{k}},
\end{aligned}
$$

and this limit exists at no point of $\left(0 \leqq x_{k} \leqq 1, k=1, \cdots, q\right)$. Thus if 0 $\leqq v\left(m_{k}\right)=o\left(\left(\log \log m_{k}\right)^{2}\right) \quad(k=1, \cdots, q), v\left(m_{k}\right) \rightarrow \infty$ and is nondecreasing and $\sum b_{m} v\left(m_{1}\right) \cdots v\left(m_{q}\right)<\infty$, then there exists an orthogonal series $\sum b_{m} \psi_{m}$ which is $(C, 1, \cdots, 1)$ (rectangular) summable at no point of $\left(0 \leqq x_{k} \leqq 1\right.$, $k=1, \cdots, q$ ). (Note that we proved elsewhere that the convergence of the series $\sum[\log (m+1)]^{1+\epsilon} a_{m n}^{2}, \quad \sum[\log (n+1)]^{1+\epsilon} a_{m n}^{2}(\epsilon>0)$ and $\sum^{\prime}[\log \log m$ $\log \log n]^{2} a_{m n}^{2}$ imply the $(C, 1,1)$ summability a.e. in $E$ of the double orthogonal series $\sum a_{m n} \phi_{m n}[13]$.)

We can easily get a counter-example for $(C, 1)$ (spherical) summability if $q \geqq 4$, in which case every integer can be represented as the sum of $q$ squares of integers [8]. For each $\nu(\nu=0,1,2, \cdots)$ arrange the solutions of $\sum m_{k}^{2}=\nu$ in some systematic order and let $\left(n_{1}, \cdots, n_{q}\right)$ be the first solution of the set. Set $\psi_{n_{1}} \cdots n_{q}(P)=\psi_{\nu}\left(x_{q}\right) \psi_{0}\left(x_{1}\right) \cdots \psi_{0}\left(x_{q-1}\right)$ and choose the remaining $\psi_{m}=\psi_{m_{1}}\left(x_{1}\right) \cdots \psi_{m_{q}}\left(x_{q}\right)\left(m_{k} \neq n_{k}, k=1, \cdots, q\right)$ in some arbitrary but orderly fashion so that every set of subscripts $\left(m_{1}, \cdots, m_{q}\right)$ satisfying $\sum m_{k}^{2}=\nu$ occurs once and only once. Let $b_{n_{1}} \cdots n_{q}=b_{\nu}$ and all other $b_{m_{1} \cdots m_{q}}$ $=0$. Let $v(\nu)$ be any positive nondecreasing monotonic sequence approaching infinity with $\nu$ and such that $v(\nu)=o\left((\log \log \nu)^{2}\right)$. Then $v(\nu)$ satisfies the hypotheses of the theorem quoted for $q=1$. Also $\sum_{m_{1}, \ldots, m_{q}=0}^{\infty} b_{m}^{2} v(\nu)=\sum_{\nu=0}^{\infty} b_{\nu} v(\nu)$ 
$<\infty$, but

$$
\begin{aligned}
\sigma_{N} & =\sum_{\nu=0}^{N}\left(1-\frac{\nu}{N+1}\right) \sum_{\Sigma_{m}=\nu} b_{m} \psi_{m}(P) \\
& =\sum_{\nu=0}^{N}\left(1-\frac{\nu}{N+1}\right) b_{n_{1} \cdots n_{q}} \psi_{n_{1} \cdots n_{q}}(P) \\
& =\psi_{0}\left(x_{1}\right) \cdots \psi_{q-1}\left(x_{q-1}\right) \sum_{\nu=0}^{N}\left(1-\frac{\nu}{N+1}\right) b_{\nu} \psi_{\nu}\left(x_{q}\right)
\end{aligned}
$$

approaches a limit at no point of $\left(0 \leqq x_{k} \leqq 1, k=1, \cdots, q\right)$. Thus for all $q \geqq 4$ if $v(\nu)=o\left((\log \log \nu)^{2}\right), 0 \leqq v(\nu) \rightarrow \infty$ as $\nu \rightarrow \infty$ and is nondecreasing and $\sum b_{m} v(\nu)<\infty$, then there exists an orthogonal series $\sum b_{m} \psi_{m}(P)$ which is $(C, 1)$ (spherically) summable at no point of $\left(0 \leqq x_{k} \leqq 1, k=1, \cdots, q\right)$.

4.4. Equivalence of the summability methods. We prove the following important result.

THEOREM 4.3. For any multiple orthogonal series $\sum a_{m} \phi_{m}$ the Cesàro summability methods $(C, \alpha)(\alpha>0)$, the Poisson-Abel method, and the typical Riesz mean summability $\left(R, \lambda_{\nu}, \alpha\right)(\alpha>0)$, where $\lambda_{\nu}$ is a logarithmico-exponential function of $\nu$ such that $\lambda_{\nu}=O\left(\nu^{c}\right)$ (c a constant), are equivalent.

A multiple orthogonal series is said to be $\left(R, \lambda_{\eta}, \alpha\right)$ summable if $S_{R}$ $=\sum_{\lambda_{\nu} \leqq R}\left(1-\lambda_{\nu} / R\right)^{\alpha} 0_{\nu}(0<R<\infty)$ approaches a limit as $R \rightarrow \infty$, and PoissonAbel summable if $\sum x^{\nu} 0$, is convergent for $0 \leqq x<1$ and $\lim _{x \rightarrow 1} \sum_{\nu=0}^{\infty} x^{\nu} 0_{\nu}$ exists $\left({ }^{8}\right)$.

The proof of the theorem is a consequence of the following set of lemmas [cf. 12]:

(i) $\lim _{N \rightarrow \infty}(N+1)^{-1} \sum_{k=0}^{N}\left(\sigma_{k}^{\alpha}-\sigma_{k}^{\alpha-1}\right)^{2}=0$ for $\alpha>1 / 2$, where $\sigma_{k}^{\alpha}$ is defined by (1.5).

Proof. Now

$$
\sigma_{k}^{\alpha}-\sigma_{k}^{\alpha-1}=-\left(\alpha p_{k}^{\alpha}\right)^{-1} \sum_{j=0}^{k} j p_{k-j}^{\alpha-1} 0_{j}
$$

Also, by the $\mathrm{ON}$ relations

$$
\int_{E}\left(\sigma_{k}^{\alpha}-\sigma_{k}^{\alpha-1}\right)^{2} d x=\left(p_{k}^{\alpha}\right)^{-2} \sum_{j=0}^{k} \alpha^{-2} j^{2}\left(p_{k-j}^{\alpha-1}\right)^{2} A_{j}
$$

and setting

$$
\delta_{N}^{\alpha}=(N+1)^{-1} \sum_{k=0}^{N}\left(\sigma_{k}^{\alpha}-\sigma_{k}^{\alpha-1}\right)^{2}
$$

we have

(8) From the second property of $\$ 2.1$ the series $\sum_{v=0}^{\infty} 0_{\nu} x^{\nu}$ is convergent for $|x|<1$. 


$$
\begin{aligned}
\int_{E} \delta_{2^{N}}^{\alpha} d x & =\alpha^{-2}\left(2^{N}+1\right)^{-1} \sum_{k=0}^{2 N}\left(p_{k}^{\alpha}\right)^{-2} \sum_{j=0}^{k} j^{2}\left(p_{k-j}^{\alpha-1}\right)^{2} A_{j} \\
& =\alpha^{-2}\left(2^{N}+1\right)^{-1} \sum_{j=0}^{2 N} j^{2} A_{j} \sum_{k=j}^{2 N}\left(p_{k}^{\alpha}\right)^{-2}\left(p_{k-j}^{\alpha-1}\right)^{2}
\end{aligned}
$$

Since it is well known that

$$
\begin{aligned}
\sum_{k=j}^{\infty}\left(p_{k}^{\alpha}\right)^{-2}\left(p_{k-j}^{\alpha-1}\right)^{2} & =O\left(j^{-1}\right) \text { if } \alpha>1 / 2 \\
\int_{E} \delta_{2}^{\alpha} d x & =O\left(2^{-N} \sum_{j=0}^{2 N} j A_{j}\right)
\end{aligned}
$$

and

$$
\begin{aligned}
\sum_{N=0}^{\infty} \int_{E} \delta_{2^{N}}^{\alpha} d x & =O\left(\sum_{N=0}^{\infty} 2^{-N} \sum_{j=0}^{2 N} j A_{j}\right) \\
& =O\left(\sum_{j=1}^{\infty} j A_{j} \sum_{N=E[\log j]} 2^{-N}\right) \\
& =O\left(\sum a_{m}^{2}\right) .
\end{aligned}
$$

Therefore $\lim _{N \rightarrow \infty} \delta_{2^{N}}^{\alpha}=0$ a.e. Also, for $2^{N}<k<2^{N+1}, 0 \leqq \delta_{k}^{\alpha} \leqq 2 \delta_{2}^{\alpha+1}$ and consequently (i) is proved.

(ii). If a multiple orthogonal series is $(C, \alpha)$ summable $(\alpha>1 / 2)$ in $E$ to $S$, then a.e. in $E$,

$$
\lim _{N \rightarrow \infty}(N+1)^{-1}\left(\left|S-\sigma_{0}^{\alpha-1}\right|+\cdots+\left|S-\sigma_{N}^{\alpha-1}\right|\right)=0
$$

and

$$
\lim _{N \rightarrow \infty}(N+1)^{-1}\left[\left(S-\sigma_{0}^{\alpha-1}\right)^{2}+\cdots+\left(S-\sigma_{N}^{\alpha-1}\right)^{2}\right]=0 .
$$

For any sequence, $\lim _{N \rightarrow \infty} \sigma_{N}^{\alpha}=S$ implies $\lim _{N \rightarrow \infty}(N+1)^{-1} \sum_{k=0}^{N}\left(\sigma_{k}^{\alpha}-S\right)^{2}$ $=0$. Relation (4.2) follows from this result and (i) by Minkowski's inequality, and (4.1) results from (4.2) by the Schwarz inequality.

(iii) The series $\sum_{n=1}^{\infty} n\left(\sigma_{n}-\sigma_{n-1}\right)^{2}<\infty$ a.e. in $E$, where $\sigma_{n}=\sigma_{n}^{1}$.

Proof. For $n \geqq 1$ by the $\mathrm{ON}$ properties

$$
\begin{aligned}
\int_{E}\left(\sigma_{n}-\sigma_{n-1}\right)^{2} d x & =\int_{E}\left(\sum_{\nu=0}^{n} \nu n^{-1}(n+1)^{-1} 0_{\nu}\right)^{2} d x \\
& =\sum_{\nu=0}^{n} \nu^{2} n^{-2}(n+1)^{-2} A_{\nu}
\end{aligned}
$$


Also,

$$
\begin{aligned}
\sum_{n=1}^{\infty} n \int_{E}\left(\sigma_{n}-\sigma_{n-1}\right)^{2} d x & \leqq \sum_{n=1}^{\infty} n^{-3} \sum_{\nu=1}^{n} \nu^{2} A_{\nu} \\
& =\sum_{\nu=1}^{\infty} \nu^{2} A_{\nu} \sum_{n=\nu}^{\infty} n^{-3} \leqq \sum a_{m}^{2}<\infty,
\end{aligned}
$$

from which (iii) follows.

(iv). For any multiple orthogonal series, $(C, 1)$ summability and PoissonAbel summability are equivalent.

The proof of (iv) depends only on (iii) and certain theorems for simple series so that the proof given in $[12$, p. 189] is valid here.

(v) For any multiple orthogonal series, $(C, 1)$ summability and $(C, \alpha)$ $(\alpha>0)$ summability are equivalent.

As for (iv) the proof in [12, pp. 189-190] may be used here.

The remainder of Theorem 4.3 follows from the fact already pointed out that $(R, \nu, \alpha)$ summability is equivalent to $(C, \alpha)$ summability for $\alpha>0$, and the result due to Hardy [7] that summability $(R, \nu, \alpha)$ implies summability $\left(R, \lambda_{\nu}, \alpha\right)(\alpha>0)$ if $\lambda_{\nu}$ is a logarithmico-exponential function of $\nu-\lambda_{\nu}=O\left(\nu^{c}\right)$, where $c$ is a constant.

REMARK. The following theorems analogous to Theorem 4.3 have been proved for multiple Fourier series:

(a) Let $f$ be summable in $\left(0 \leqq x_{j} \leqq 2 \pi, j=1, \cdots, q\right)$ and periodic of period $2 \pi$ for each variable. If $f \geqq 0$ in the neighborhood of a point $Y$, then the Fourier series of $f$ at $Y$ is either summable by every Riesz mean of order greater than $(q-1) / 2$ or summable by no Riesz mean. The necessary and sufficient condition for summability to sum $S 2^{q / 2-1} \Gamma(q / 2)$ is that $\lim _{T \rightarrow 0} T^{-q} \int_{0}^{T}\left(f_{Y}(t)-S\right) t^{q-1} d t=0$, where $f_{Y}(t)$ is the spherical mean-function of $f$ at the point $Y[6]$.

(b) If $T^{-q-2 p} \int_{0}^{T} t^{q+2 p-1}\left|f_{p}(t)\right| d t=O(1)$ as $T \rightarrow 0\left({ }^{9}\right)$, or, in particular, if $f_{p}(t)=O(1)$, then the multiple Fourier series of $f$ is either summable $(R, \nu, \alpha)$ for every $\alpha>p+(q-1) / 2$ or for no $\alpha$; a necessary and sufficient condition for it to be summable is that $f_{r}(t) \rightarrow S$ as $t \rightarrow 0$ for $r>p+1$ [5].

\section{REFERENCES}

1. R. P. Agnew, On double orthogonal series, Proc. London Math. Soc. vol. 33 (1932) pp. $420-434$.

2. S. Banach, Sur la convergence presque partout de functionnelles linéaires, Bull. Sci. Math. vol. 61 (1926) pp. 27-32, 36-43.

3. S. Bochner, Summation of multiple Fourier series by spherical means, Trans. Amer. Math. Soc. vol. 40 (1936) pp. 175-207.

4. K. Chandrasekharan, On summation of multiple Fourier series. III, Bull. Amer. Math. Soc. vol. 52 (1946) pp. 474-477.

(9) The function $f_{p}(t)$ is the spherical mean-function of order $p$ of $f$. 
5. On multiple Fourier series, Proceedings of the Indian Academy of Sciences, Section A, vol. 24 (1946) pp. 229-232.

6. M. T. Cheng, Some Tauberian theorems with application to multiple Fourier series, Ann. of Math. vol. 50 (1949) pp. 763-776.

7. G. H. Hardy, The second theorem of consistency for summable series, Proc. London Math. Soc. vol. 15 (1916-1917) pp. 72-88.

8. G. H. Hardy and E. M. Wright, An introduction to the theory of numbers, Oxford University Press, 1938.

9. G. H. Hardy, Divergent series, Oxford University Press, 1949.

10. E. Helly, Über Reihenentwicklungen nach Funktionen eines Orthogonalsystems, Akademie der Wissenschaften in Wien. Mathematisch-Naturwissenschaftliche Klasse. Sitzungsberichte. Abteilung IIa vol. 121 (1912).

11. S. Kaczmarz, Sur la convergence et la sommabilité des développements orthogonaux, Studia Mathematica vol. 1 (1929) pp. 87-121.

12. S. Kaczmarz and H. Steinhaus, Theorie der Orthogonalreihen, Monografi Matematice, vol. 6, Warsaw, 1935.

13. J. Mitchell, A summability theorem for double orthogonal series whose coefficients satisfy certain conditions, Amer. J. Math. vol. 71 (1949) pp. 257-268.

14. M. Morse and W. Transue, The Fréchet variation and the convergence of multiple Fourier series. Proc. Nat. Acad. Sci. U.S.A. vol. 35 (1949) pp. 395-399.

15. H. Rademacher, Einige Sätze über Reihen von allgemeinen Orthogonalfunktionen, Math. Ann. vol. 87 (1922) pp. 112-138.

16. A. Walfisz, Über Gitterpunkte in mehrdimensionalen Ellipsoiden, Math. Zeit. vol. 19 (1924) pp. 300-307.

\section{UNIVERSITY OF ILLINOIS}

URBANA, ILl. 